\title{
FASTER ALGORITHMS FOR MINIMUM CYCLE BASIS IN DIRECTED GRAPHS*
}

\author{
RAMESH HARIHARAN ${ }^{\dagger}$, TELIKEPALLI KAVITHA ${ }^{\ddagger}$, AND KURT MEHLHORN ${ }^{\S}$
}

\begin{abstract}
We consider the problem of computing a minimum cycle basis in a directed graph. The input to this problem is a directed graph $G$ whose edges have nonnegative weights. A cycle in this graph is actually a cycle in the underlying undirected graph with edges traversable in both directions. A $\{-1,0,1\}$ edge incidence vector is associated with each cycle: edges traversed by the cycle in the right direction get 1 and edges traversed in the opposite direction get -1 . The vector space over $\mathbb{Q}$ generated by these vectors is the cycle space of $G$. A set of cycles is called a cycle basis of $G$ if it forms a basis for this vector space. We seek a cycle basis where the sum of weights of the cycles is minimum. The current fastest algorithm for computing a minimum cycle basis in a directed graph with $m$ edges and $n$ vertices runs in $\tilde{O}\left(m^{\omega+1} n\right)$ time, where $\omega<2.376$ is the exponent of matrix multiplication. We present an $O\left(m^{3} n+m^{2} n^{2} \log n\right)$ algorithm. We obtain our algorithm by using fast matrix multiplication over rings and an efficient extension of Dijkstra's algorithm to compute a shortest cycle in $G$ whose dot product with a function on its edge set is nonzero. We also present a simple $O\left(m^{2} n+m n^{2} \log n\right)$ Monte Carlo algorithm. The problem of computing a minimum cycle basis in an undirected graph has been well studied. In this problem a $\{0,1\}$ edge incidence vector is associated with each cycle and the vector space over $\mathbb{Z}_{2}$ generated by these vectors is the cycle space of the graph. The fastest known algorithm for computing a minimum cycle basis in an undirected graph runs in $O\left(m^{2} n+m n^{2} \log n\right)$ time and our randomized algorithm for directed graphs matches this running time.
\end{abstract}

Key words. cycle basis, fast matrix multiplication, randomization, shortest paths

AMS subject classifications. 68W20,68W40

DOI. $10.1137 / 060670730$

1. Introduction. Let $G=(V, E)$ be a directed graph with $m$ edges and $n$ vertices. A cycle in $G$ is actually a cycle in the underlying undirected graph, i.e., edges are traversable in both directions. Associated with each cycle is a $\{-1,0,1\}$ edge incidence vector: edges traversed by the cycle in the right direction get 1 , edges traversed in the opposite direction get -1 , and edges not in the cycle at all get $0 .{ }^{1}$ The vector space over $\mathbb{Q}$ generated by these vectors is the cycle space of $G$. A set of cycles is called a cycle basis if it forms a basis for this vector space. When $G$ is connected, the cycle space has dimension $d=m-n+1$.

We assume that there is a weight function $w: E \rightarrow \mathbb{R}^{\geq 0}$, i.e., the edges of $G$ have nonnegative weights assigned to them. The weight of a cycle basis is the sum of the weights of its cycles. A minimum cycle basis of $G$ is a cycle basis of minimum weight. We consider the problem of computing a minimum cycle basis in a given digraph.

* Received by the editors September 26, 2006; accepted for publication (in revised form) April 24, 2008; published electronically September 8, 2008. Preliminary versions of this work appeared in $[14,16]$.

http://www.siam.org/journals/sicomp/38-4/67073.html

${ }^{\dagger}$ Strand Life Sciences, 237, Sir C. V. Raman Avenue, Sadashivnagar, Bangalore 560080, India (ramesh@strandls.com).

${ }^{\ddagger}$ Computer Science and Automation, Indian Institute of Science, Bangalore 560012, India (kavitha@csa.iisc.ernet.in).

$\S$ Max-Planck-Institut für Informatik, Stuhlsatzenhausweg 85, Saarbrücken 66123, Germany (mehlhorn@mpi-sb.mpg.de).

${ }^{1}$ Formally, the incidence vector is determined only up to a factor \pm 1 as either direction (clockwise or counterclockwise) could be chosen to traverse a cycle. 
A related problem pertains to undirected graphs, where we associate a $\{0,1\}$ edge incidence vector with each cycle; edges in the cycle get 1 and others get 0 . Unlike directed graphs where the cycle space is defined over $\mathbb{Q}$, cycle spaces in undirected graphs are defined as vector spaces over $\mathbb{Z}_{2}$. The minimum cycle basis problem in an undirected graph $G$ asks for the cycle basis of minimum weight in $G$.

The different possible settings for the minimum cycle basis problem are (i) the minimum cycle basis problem in undirected graphs, (ii) the minimum cycle basis problem in directed graphs (where the directions on the cycles are ignored), and (iii) the minimum directed cycle basis problem in directed graphs, where the cycle basis has to consist of cycles that traverse edges only along the direction of the edge.

We first compare problems (i) and (ii) and then discuss problem (iii). Problems (i) and (ii) are essentially different, since the first problem deals with computing a minimum weight spanning set of cycles that is linearly independent over $\mathbb{Z}_{2}$ while the second problem needs to compute a minimum weight spanning set of cycles that is linearly independent over $\mathbb{Q}$. Transforming cycles in a cycle basis of a directed graph by replacing both -1 and 1 by 1 does not necessarily yield a basis for the underlying undirected graph, since the given cycle basis could be linearly independent over $\mathbb{Q}$ but linearly dependent over $\mathbb{Z}_{2}$. In addition, lifting a minimum cycle basis of the underlying undirected graph by putting back directions does not necessarily yield a minimum cycle basis for the directed graph. Examples of both phenomena were presented in [22], which we include in section 2. Thus, one cannot find a minimum cycle basis for a directed graph by simply working with the underlying undirected graph.

A directed cycle basis is a spanning set of cycles where the incidence vector of each cycle in this basis is a vector in $\{0,1\}^{m}$, that is, each edge in a cycle here is traversed in the right direction. Note that a directed graph need not admit a directed cycle basis. Berge [2] studied the question of when a directed graph $G$ admits such a cycle basis. He showed that if $G$ is strongly connected, then $G$ admits a directed cycle basis. Conversely, he showed that if $G$ admits a directed cycle basis, then each maximal weakly connected induced subgraph of $G$ with no cut vertex has to be strongly connected or a single arc.

Efficient algorithms for computing minimum cycle bases in the above settings have several applications. The minimum cycle basis problem is a special case of the null space problem. The null space problem is defined as follows: given a field $\mathbb{F}$ and an $n \times m$ matrix $A$ with $n \leq m$, rank $r$, and entries in $\mathbb{F}$, find a matrix with the fewest nonzeros, whose columns span the null space of $A$. The null space problem was studied by Coleman and Pothen [6,7], and this problem is NP-hard in general. The minimum cycle basis problem in undirected graphs with unit weights on the edges arises when the underlying field is $\mathbb{Z}_{2}$ and $A$ is the $\{0,1\}$ vertex-edge incidence matrix of an undirected graph $G(V, E)$. The null vectors of this $A$ are the vectors $\left(x_{e}\right)_{e \in E} \in \mathbb{Z}_{2}^{|E|}$ such that for each $v \in V$ we have $\sum_{e \in \delta(v)} x_{e}=0(\bmod 2)$, where $\delta(v)$ is the set of edges incident on $v$. Note that the set of such vectors $\left(x_{e}\right)_{e \in E}$ is the cycle space of $G$, and a solution to the null space problem is a minimum cycle basis of $G$.

Similarly, the minimum cycle basis problem in directed graphs with unit weights on the edges is an instance of the null space problem when the underlying field is $\mathbb{Q}$ and $A$ is the $\{-1,0,1\}$ vertex-edge incidence matrix of a directed graph $G(V, E)$. The null vectors of such an $A$ are the vectors $\left(x_{e}\right)_{e \in E} \in \mathbb{Q}^{|E|}$ such that for each $v \in V$ we have $\sum_{e \in \delta^{+}(v)} x_{e}-\sum_{e \in \delta^{-}(v)} x_{e}=0$, where $\delta^{+}(v)$ and $\delta^{-}(v)$ are the set of edges leaving $v$ and entering $v$, respectively. It is easy to see that the set of such vectors $\left(x_{e}\right)_{e \in E}$ is the cycle space of $G$. The minimum cycle basis of $G$ is a solution to this 
null space problem. Indeed, assume that $B$ is a minimum solution to the null space problem. We may assume that the entries of $B$ are integral, as multiplication by a suitable constant makes all entries integral and does not change the number of nonzero entries. So assume that $B$ contains a column $C$ whose entries are not in $\{0, \pm 1\}$. Since $C$ belongs to the null space of the vertex-edge incidence matrix $A, C$ decomposes into a set of simple cycles $C_{1}$ to $C_{k}$. Each $C_{i}$ uses a subset (not necessarily proper) of the edges of $C$ and $B \backslash C \cup C_{i}$ is a basis for some $i$. We conclude that there exists a solution with $0, \pm 1$ entries to this null space problem, which implies that a minimum cycle basis of the directed graph $G$ is a solution to this null space problem.

Some of the applications of minimum cycle bases arise from the above characterization of minimum cycle bases as the solutions of the null space problem in graphs. The cycle analysis of electrical networks [9] corresponding to Kirchoff's law is such an example. Applications of minimum cycle bases have also been shown in structural engineering [5], chemistry and biochemistry [11, 19], and surface reconstruction from point clouds [23]. The minimum directed cycle basis has applications in metabolic flux analysis [12]. A cycle basis of minimum weight in a directed graph whose $d \times m$ cycle-edge incidence matrix satisfies the constraint that all its regular $d \times d$ submatrices have determinant \pm 1 has been found to be very useful in cyclic timetabling $[20,21]$. Books by Deo [10] and Bollobás [4] have in-depth coverage of cycle bases.

Horton [15] designed the first polynomial time algorithm to compute a minimum cycle basis in an undirected graph and there are now several polynomial time algorithms for this problem $[3,9,13,18]$, the fastest running in $O\left(m^{2} n+m n^{2} \log n\right)$ time [18]. Gleiss, Leydold, and Stadler [12] used Berge's characterization of directed cycle bases and showed that a generalization of Horton's minimum cycle basis algorithm in undirected graphs computes a minimum directed cycle basis in strongly connected directed graphs. The first polynomial time algorithm for computing a minimum cycle basis in a directed graph had a running time of $\tilde{O}\left(m^{4} n\right)$ [17]. Liebchen and Rizzi [22] gave an $\tilde{O}\left(m^{\omega+1} n\right)$ algorithm for this problem, where $\omega<2.376$ [8] is the exponent of matrix multiplication; this was the fastest deterministic algorithm so far for this problem in directed graphs.

In this paper we present an $O\left(m^{3} n+m^{2} n^{2} \log n\right)$ deterministic algorithm and an $O\left(m^{2} n+m n^{2} \log n\right)$ Monte Carlo algorithm to compute a minimum cycle basis in a directed graph $G$ with $m$ edges, $n$ vertices, and nonnegative edge weights. The running time of our deterministic algorithm is $m$ times the running time of the fastest algorithm for computing minimum cycle bases in undirected graphs, we leave it as a challenge to close the gap. The increased complexity seems to stem from the larger base field. Arithmetic in $\mathbb{Z}_{2}$ suffices for undirected graphs. For directed graphs, the base field is $\mathbb{Q}$, which seems to necessitate the handling of large numbers. Also, the computation of a shortest cycle that has a nonzero dot product with a given vector seems more difficult in directed graphs than in undirected graphs.

The framework used in our algorithms was introduced by de Pina [9] and was also used in $[3,18,17]$ : we compute cycles $C_{i}$ and supporting vectors $N_{i}$ so that each $C_{i}$ is a shortest cycle not orthogonal to its corresponding $N_{i}$, and each $N_{i}$ is orthogonal to all previous $C_{j}, j<i$. This collection of cycles $C_{i}$ is known to be a minimum cycle basis. Our algorithms for computing the $C_{i}$ 's and $N_{i}$ 's rest on two ideas.

First, we show how to compute the vectors $N_{i}$ efficiently using fast matrix multiplication and inversion. Our basic algorithm updates all vectors $N_{j}$ with $j>i$ in iteration $i$, which results in an $\tilde{O}\left(m^{4}\right)$ algorithm. The improvement rests on an idea already used in [18] to delay the update of vectors with higher index and to perform these updates in bulk using matrix multiplication and inversion. However, this creates 
a problem since the numbers involved in the arithmetic get very large. We show two approaches to solving this problem, leading to an $O\left(m^{2} n+m n^{2} \log n\right)$ randomized algorithm and an $O\left(m^{3} n+m^{2} n^{2} \log n\right)$ deterministic algorithm. In the randomized algorithm we work over a finite field $\mathbb{Z}_{p}$ for a small prime $p$, which ensures that the numbers involved here do not get large. We show that we compute a minimum cycle basis with a probability of at least $3 / 4$ when $p$ is a prime chosen uniformly at random from a set of $d^{2}$ small primes. In the deterministic algorithm, we could run into intermediate numbers whose bit size is $\tilde{\Theta}\left(m^{2}\right)$ when we use fast matrix inversion algorithms. This makes the running time of our algorithm $\tilde{\Theta}\left(m^{\omega+2}\right)$, which is worse than the original iterative algorithm. We circumvent the problem by working over a suitable ring $\mathbb{Z}_{R}$. The important point is that there is no fixed $R$ over which we work during the entire algorithm: whenever we perform the matrix multiplication and inversion, we determine a suitable $R$ so that the inverse of the matrix that we seek to invert exists in $\mathbb{Z}_{R}$. This leads us to the vectors $N_{i} \bmod R$, and the number $R$ will be large enough so that we can easily recover the original vectors $N_{i}$ from $N_{i} \bmod R$. The total time needed now is $\tilde{O}\left(m^{\omega+1}\right)$.

The second key step in our algorithm is a subroutine to compute a shortest cycle whose dot product with a given vector $N_{i}$ is nonzero modulo a small number $p$. We present an $O\left(m n+n^{2} \log n\right)$ algorithm to compute such a cycle $C_{p}$. This algorithm is obtained by computing two types of paths between each adjacent pair of vertices. The first path is a shortest path between these two vertices and the second is a shortest path whose residue class is different from the residue class of the first path. Thus this yields an $O\left(m^{2} n+m n^{2} \log n\right)$ algorithm over $\mathbb{Z}_{p}$ for computing the $d$ cycles. For the deterministic algorithm, the computation of each cycle can be reduced via the Chinese remainder theorem to computing a shortest cycle $C_{p}$ whose inner product with $N_{i}$ is nonzero modulo $p$ for some $p \in\left\{p_{1}, \ldots, p_{d}\right\}$, which is a collection of small primes. This procedure is repeated for each $p \in\left\{p_{1}, \ldots, p_{d}\right\}$ yielding $O\left(m^{2} n+m n^{2} \log n\right)$ time for each $C_{i}$ and thus $O\left(m^{3} n+m^{2} n^{2} \log n\right)$ time overall for all of the $d$ cycles.

Organization of this paper. In section 2 we discuss some preliminaries and describe the examples from [22] that were mentioned in section 1. Our framework is given in section 3, and we present a simple deterministic algorithm from [17] that follows from this framework. Section 4 lays the approach for a faster scheme and shows the problem of large numbers that such an approach runs into. Section 5 describes the deterministic algorithm that overcomes this problem, and section 6 gives a randomized algorithm. Section 7 describes the subroutine for computing the required cycles.

2. Preliminaries. We are given a digraph $G=(V, E)$, where $|V|=n$ and $|E|=$ $m$. Without loss of generality, the underlying undirected graph of $G$ is connected. Then $d=m-n+1$ is the dimension ${ }^{2}$ of the cycle space of $G$. So a minimum cycle basis of $G$ consists of $d$ cycles $C_{1}, \ldots, C_{d}$. We describe cycles by their incidence vectors in $\{-1,0,+1\}^{m}$.

A cycle basis of a directed graph need not project onto an undirected cycle

\footnotetext{
${ }^{2}$ Fix any spanning tree of $G$. For a nontree edge $e$, the fundamental cycle $F_{e}$ induced by $e$ consists of $e$ plus the tree path connecting its endpoints. This set of cycles is clearly independent as every nontree edge is contained in a single cycle. We need to show that it spans all cycles. Consider any cycle $C=\left(x_{e}\right)_{e \in E}$ and define the sum $S=\sum_{e}$ is a nontree edge $x_{e} F_{e}$, in which every fundamental cycle is multiplied with the multiplicity of its defining edge in $C$. The vector $S$ is in the cycle space and so is $Z=S-C$. The entries of $Z$ corresponding to nontree edges are zero (by the definition of $S$ ) and $Z$ satisfies the flow conservation constraints. Hence the entries of $Z$ corresponding to tree edges must also be zero. Thus $Z=0$ and the fundamental cycles form a basis. The number of fundamental cycles is exactly $m-n+1$.
} 
basis. Consider the following three 4-cycles in the directed graph in Figure 2.1: $C_{1}=\left(e_{1}, e_{2}, e_{3}, e_{4}\right), C_{2}=\left(e_{1}, e_{6}, e_{3}, e_{5}\right)$, and $C_{3}=\left(e_{2}, e_{5}, e_{4}, e_{6}\right)$ given by the vectors $(1,1,1,1,0,0),(1,0,-1,0,-1,-1)$, and $(0,1,0,-1,-1,1)$. It is easy to see that these vectors are linearly independent over $\mathbb{Q}$. Hence they form a cycle basis for the directed $K_{4}$. But in the underlying undirected graph, each of these cycles is equal to the sum modulo 2 of the other two, so $C_{1}, C_{2}, C_{3}$ do not form a cycle basis for the undirected $K_{4}$.
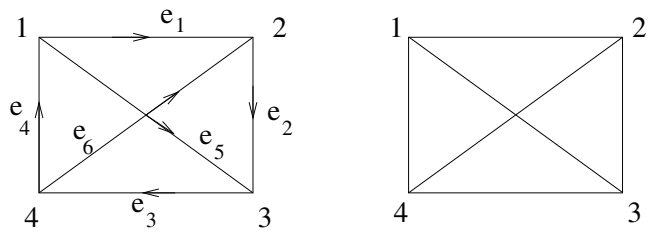

FIG. 2.1. Directed $K_{4}$ and the underlying undirected graph.

Further, there are directed weighted graphs in which the minimum cycle basis has lower weight than any cycle basis of the underlying undirected graph; such an example was given in [22]. Consider the generalized Petersen graph $P_{7,2}$ in Figure 2.2.

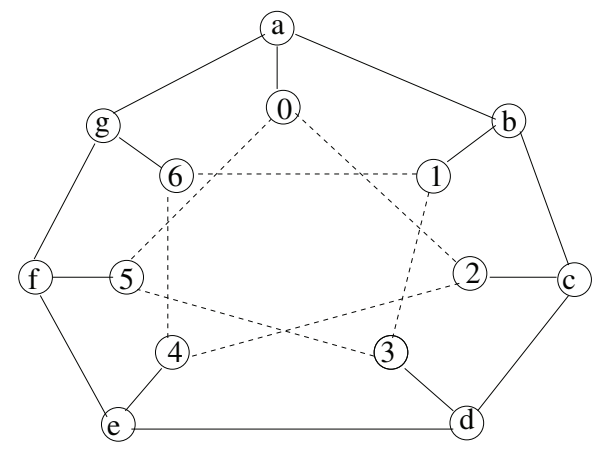

FIG. 2.2. The generalized Petersen graph $P_{7,2}$.

Call an edge $(u, v)$ an inner edge if $\{u, v\} \subset\{0,1, \ldots, 6\}$. Similarly call an edge $(u, v)$ an outer edge if $\{u, v\} \subset\{a, \ldots, g\}$. The seven edges that remain are called spokes. Assign weight two to the seven inner edges and weight three to the outer edges and spokes. The shortest cycle in this graph has a weight of 14 and there are precisely eight cycles having a weight of 14 , namely the cycle $C_{I}$ consisting of only inner edges, and the seven cycles using one inner edge, two spokes, and two outer edges. Use $C_{i}, 0 \leq i \leq 6$, to denote the cycle using the inner edge connecting $i$ and $i+2 \bmod 7$. Every other cycle has a length of at least 15 .

Every edge of $P_{7,2}$ belongs to precisely two of the eight cycles with a weight of 14 . Therefore in the undirected case, these $8=m-n+1$ cycles are not independent over $\mathbb{Z}_{2}$. Thus in the undirected case, every cycle basis has a weight of at least 113 . In the directed case, these 8 cycles under any orientation of edges are linearly independent. ${ }^{3}$ So there is a directed cycle basis of weight 112 .

\footnotetext{
${ }^{3}$ Direct the inner edges clockwise, the spokes outward, and the outer edges counterclockwise. Then all eight cycles use their inner and outer edges in the forward direction. Assume $\alpha_{I} C_{I}+$ $\sum_{0 \leq i \leq 6} \alpha_{i} C_{i}=0$. The edge $(i, i+2 \bmod 7)$ is used only by $C_{I}$ and $C_{i}$, and hence we must have $\alpha_{i}=-\alpha_{I}$ for all $i$. But then the outer edges do not cancel out.
} 
We will assume for the rest of this paper that the edges in $E=\left\{e_{1}, \ldots, e_{m}\right\}$ are ordered so that edges $e_{d+1}$ to $e_{m}$ form the edges of a spanning tree $T$ of the underlying undirected graph. This means that the first $d$ coordinates, of each of $C_{1}, \ldots, C_{d}$, correspond to edges outside the tree $T$, and the last $n-1$ coordinates are the edges of $T$. This will be important in our proofs in section 3. We can also assume that there are no multiple edges in $G$. It is easy to see that whenever there are two edges from $u$ to $v$, the heavier edge (call it $a$ ) can be deleted from $E$, and the least weight cycle (call it $C(a)$ ) that contains the edge $a$ can be added to the minimum cycle basis computed on ( $V, E \backslash\{a\})$. The cycle $C(a)$ consists of the edge $a$ and the shortest path between $u$ and $v$ in the underlying undirected graph. All such cycles can be computed by an all-pairs-shortest-paths computation in the underlying undirected graph of $G$, which takes $\tilde{O}(m n)$ time. So we will assume from now on that $m \leq n^{2}$.

3. Framework and a simple algorithm. We begin with a structural characterization of a minimum cycle basis. This characterization uses auxiliary rational vectors $N_{1}, \ldots, N_{d}$ which serve as a scaffold for proving properties of $C_{1}, \ldots, C_{d}$, as described below. We use $\left\langle v_{1}, v_{2}\right\rangle$ to denote the standard inner product or dot product of the vectors $v_{1}$ and $v_{2}$.

THEOREM 3.1. Cycles $C_{1}, \ldots, C_{d}$ form a minimum cycle basis if there are vectors $N_{1}, \ldots, N_{d}$ in $\mathbb{Q}^{m}$ such that for all $i, 1 \leq i \leq d$ :

1. Prefix orthogonality: $\left\langle N_{i}, C_{j}\right\rangle=0$ for all $j, 1 \leq j<i$.

2. Nonorthogonality: $\left\langle N_{i}, C_{i}\right\rangle \neq 0$.

3. Shortness: $C_{i}$ is a shortest cycle with $\left\langle N_{i}, C_{i}\right\rangle \neq 0$.

Proof. First, we show that $C_{1}, \ldots, C_{d}$ is a cycle basis of $G$ by showing that these are linearly independent over $\mathbb{Q}$ (recall that any set of $d$ linearly independent cycles is a cycle basis). Suppose it is not. Then a rational linear combination of a subset of these cycles yields 0 . Let the cycle with the largest index in this subset be $C_{i}$. By properties 1 and 2 of the theorem, taking the dot product of this linear combination with $N_{i}$ yields a nonzero value on one side of this linear combination and a 0 on the other side, a contradiction.

Second, we show that $C_{1}, \ldots, C_{d}$ is a minimum cycle basis of $G$. Suppose it is not. Then consider the smallest $i \geq 1$ such that $C_{1}, \ldots, C_{i}$ are not in any minimum cycle basis. Then $C_{1}, \ldots, C_{i-1}$ belong to some minimum cycle basis; call this basis $\mathcal{K}$ (in the event that $i=1$, let $\mathcal{K}$ be any minimum cycle basis). We will exhibit a cycle $K \in \mathcal{K}$ such that (i) $\left\langle N_{i}, K\right\rangle \neq 0$ and (ii) $K$ can be written as a rational linear combination of $C_{i}$ along with cycles in $\mathcal{K} /\{K\}$. Demonstrating such a cycle $K \in \mathcal{K}$ is easy: since $\mathcal{K}$ is a basis not containing $C_{i}$, the cycle $C_{i}$ must be a rational linear combination of cycles in $\mathcal{K}$. At least one of these cycles $K \in \mathcal{K}$ satisfies $\left\langle N_{i}, K\right\rangle \neq 0$, because $\left\langle N_{i}, C_{i}\right\rangle \neq 0$ by property 2 in the theorem. Therefore (i) holds. Further, (ii) follows by rewriting the above linear combination to switch the sides of $C_{i}$ and $K$.

Property 1 of the theorem and condition (i) ensure that the cycle $K$ is not one of $C_{1}, \ldots, C_{i-1}$. Condition (ii) implies that $\mathcal{K} /\{K\} \cup\left\{C_{i}\right\}$ is a cycle basis. Property 3 of the theorem and condition (i) imply that $C_{i}$ has weight at most that of $K$ and therefore $\mathcal{K} /\{K\} \cup\left\{C_{i}\right\}$ is also a minimum cycle basis. $C_{1}, \ldots, C_{i}$ belong to this minimum cycle basis, a contradiction.

We present a simple deterministic algorithm from [17] that computes $N_{i}$ 's and $C_{i}$ 's satisfying the criteria in Theorem 3.1.

The algorithm deterministic- $M C B$.

1. Initialize the vectors $N_{1}, \ldots, N_{d}$ of $\mathbb{Q}^{m}$ to the first $d$ vectors $e_{1}, \ldots, e_{d}$ of the standard basis of $\mathbb{Q}^{m}$.

(The vector $e_{i}$ has 1 in the $i$ th position and 0's elsewhere.) 
2. For $i=1$ to $d$ do

- compute $C_{i}$ to be a shortest cycle such that $\left\langle C_{i}, N_{i}\right\rangle \neq 0$,

- for $j=i+1$ to $d$ do

$$
\begin{gathered}
\text { update } N_{j} \text { as: } N_{j}=N_{j}-N_{i} \frac{\left\langle C_{i}, N_{j}\right\rangle}{\left\langle C_{i}, N_{i}\right\rangle}, \\
\text { normalize } N_{j} \text { as: } N_{j}=N_{j} \frac{\left\langle C_{i}, N_{i}\right\rangle}{\left\langle C_{i-1}, N_{i-1}\right\rangle} .
\end{gathered}
$$

(We take $\left\langle C_{0}, N_{0}\right\rangle=1$.)

The above algorithm needs the vector $N_{i}$ in the $i$ th iteration to compute the cycle $C_{i}$. Instead of computing $N_{i}$ from scratch in the $i$ th iteration, it obtains $N_{i}$ by update and normalization steps through iterations 1 to $i-1$. We describe how to compute a shortest cycle $C_{i}$ such that $\left\langle C_{i}, N_{i}\right\rangle \neq 0$ in section 7 . Let us now show that the $N_{i}$ 's obey the prefix orthogonality property. Lemma 3.2, proved in [17], shows this and more.

Lemma 3.2. For any $i$, at the end of iteration $i-1$, the vectors $N_{i}, \ldots, N_{d}$ are orthogonal to $C_{1}, \ldots, C_{i-1}$, and, moreover, for any $j$ with $i \leq j \leq d, N_{j}=$ $\left\langle N_{i-1}, C_{i-1}\right\rangle\left(x_{j, 1}, \ldots, x_{j, i-1}, 0, \ldots, 0,1,0, \ldots, 0\right)$, where 1 occurs in the $j$ th coordinate and the vector $\mathbf{x}=\left(x_{j, 1}, \ldots, x_{j, i-1}\right)$ is the unique solution to the set of equations:

$$
\left(\begin{array}{c}
\tilde{C}_{1}^{T} \\
\vdots \\
\tilde{C}_{i-1}^{T}
\end{array}\right) \mathbf{x}=\left(\begin{array}{c}
-c_{1 j} \\
\vdots \\
-c_{(i-1) j}
\end{array}\right) .
$$

Here $\tilde{C}_{k}, 1 \leq k<i$, is the restriction of $C_{k}$ to its first $i-1$ coordinates and $c_{k j}$ is the jth coordinate of $C_{k}$.

Proof. The claim is certainly true after the 0th iteration, that is, at the beginning of the algorithm. So consider the $i$ th iteration and assume that the claim is true at the end of iteration $i-1$. In iteration $i$, we determine $C_{i}$ with $\left\langle C_{i}, N_{i}\right\rangle \neq 0$ and then update the $N_{j}$ 's for all $j$ with $i+1 \leq j \leq n$. Consider any $j$ with $i+1 \leq j \leq n$ and use $N_{j}^{\prime}$ to denote the updated value of $N_{j}$.

$N_{j}$ is updated by subtracting a scalar multiple of $N_{i}$ from it. Since $N_{j}$ and $N_{i}$ are orthogonal to $C_{l}$ for $l<i$ by induction hypothesis, $N_{j}^{\prime}$ is orthogonal to $C_{l}$. The update step also guarantees orthogonality to $C_{i}$. Indeed,

$$
\left\langle C_{i}, N_{j}^{\prime}\right\rangle=\left\langle C_{i}, N_{j}\right\rangle-\left\langle C_{i}, N_{i}\right\rangle \frac{\left\langle C_{i}, N_{j}\right\rangle}{\left\langle C_{i}, N_{i}\right\rangle}=0 .
$$

By induction hypothesis, $N_{j}$ is of the form $\left(t_{j, 1}, \ldots, t_{j, i-1}, 0, \ldots, t_{j, j}, 0, \ldots\right)$, where $t_{j, j}=\left\langle C_{i-1}, N_{i-1}\right\rangle$ and $N_{i}$ has nonzero entries only in its first $i$ coordinates. So $N_{j}^{\prime}$ has the form $\left(t_{j, 1}^{\prime}, \ldots, t_{j, i}^{\prime}, 0, \ldots, t_{j, j}, 0, \ldots\right)$. After normalization, the $j$ th coordinate of $N_{j}^{\prime}$ is

$$
t_{j, j} \frac{\left\langle N_{i}, C_{i}\right\rangle}{\left\langle N_{i-1}, C_{i-1}\right\rangle}=\left\langle N_{i-1}, C_{i-1}\right\rangle \frac{\left\langle N_{i}, C_{i}\right\rangle}{\left\langle N_{i-1}, C_{i-1}\right\rangle}=\left\langle N_{i}, C_{i}\right\rangle
$$

Hence $N_{j}^{\prime}$ has the form

$$
N_{j}^{\prime}=\left\langle N_{i}, C_{i}\right\rangle\left(u_{j, 1}, \ldots, u_{j, i}, 0, \ldots, 1,0, \ldots, 0\right) .
$$


Since $N_{j}^{\prime}$ is orthogonal to $C_{1}, \ldots, C_{i}$ and $\left\langle N_{i}, C_{i}\right\rangle \neq 0,\left(u_{j, 1}, \ldots, u_{j, i}\right)$ is a solution to the following set of equations:

$$
\left(\begin{array}{c}
\tilde{C}_{1}^{T} \\
\vdots \\
\tilde{C}_{i}^{T}
\end{array}\right) \mathbf{x}=\left(\begin{array}{c}
-c_{1 j} \\
\vdots \\
-c_{i j}
\end{array}\right),
$$

where $\tilde{C}_{k}$, for $k=1, \ldots, i$, is the restriction of the vector $C_{k}$ to its first $i$ coordinates and $c_{k j}$ is the $j$ th coordinate of the vector $C_{k}$. We show that the matrix of $\tilde{C}_{k}$ 's is nonsingular, hence $\left(u_{j, 1}, \ldots, u_{j, i}\right)$ is the unique solution of (3.2). The proof of the nonsingularity of this matrix mimics the argument in Theorem 3.1. Consider any linear combination of the rows adding to the zero vector:

$$
\sum_{k=1}^{i} \alpha_{k} \tilde{C}_{k}=\mathbf{0} .
$$

Assume that one of the $\alpha_{k}$ 's is nonzero and consider the largest $\ell$ such that $\alpha_{\ell} \neq 0$. We take the inner product of both sides of (3.3) with $\tilde{N}_{\ell}$, where $\tilde{N}_{\ell}$ is the restriction of the vector $N_{\ell}$ to its first $i$ coordinates. Note that $\tilde{N}_{\ell}$ has all of the nonzero entries of $N_{\ell}$ since $\ell \leq i$ and only the first $\ell$ entries of $N_{\ell}$ may be nonzero. So $\left\langle\tilde{C}_{k}, \tilde{N}_{\ell}\right\rangle=\left\langle C_{k}, N_{\ell}\right\rangle$ for all $k \leq \ell$. Hence the left-hand side is $\sum_{k=1}^{\ell} \alpha_{k}\left\langle C_{k}, N_{\ell}\right\rangle=\alpha_{\ell}\left\langle C_{\ell}, N_{\ell}\right\rangle$ since $\left\langle C_{k}, N_{\ell}\right\rangle=0$ for each $k$ with $k<\ell$. Since $\alpha_{\ell}$ and $\left\langle C_{\ell}, N_{\ell}\right\rangle$ are nonzero while the right-hand side is zero, we get a contradiction. Thus all of the $\alpha_{k}$ 's in (3.3) are zero and so the matrix with $\tilde{C}_{k}$ 's as its rows is nonsingular and the proof is complete.

Remark. Note that the $i$ th coordinate of $N_{i}$ is nonzero. This readily implies that there is at least one cycle that has nonzero dot product with $N_{i}$, namely the fundamental cycle $F_{e_{i}}$ formed by the edge $e_{i}$ and the path in the spanning tree $T$ connecting its endpoints. The dot product $\left\langle F_{e_{i}}, N_{i}\right\rangle$ is equal to the $i$ th coordinate of $N_{i}$, which is nonzero.

We next give an alternative characterization of these $N_{j}$ 's. This characterization helps us in bounding the running time of the algorithm deterministic-MCB. Let $M$ denote the $(i-1) \times(i-1)$ matrix of $\tilde{C}_{k}$ 's in $(3.1)$, and let $b_{j}$ denote the column vector of $-c_{k j}$ 's on the right. We claim that solving $M \mathbf{x}=\operatorname{det}(M) \cdot b_{j}$ leads to the same vectors $N_{j}$ for all $j$ with $i \leq j \leq d$. We first show the following claim.

LEMMA 3.3. $\left\langle N_{i-1}, C_{i-1}\right\rangle=\operatorname{det}(M)$.

Proof. Let $X$ be the $(i-1) \times(i-1)$ matrix with its $k$ th column equal to $N_{k}$ truncated to its first $i-1$ coordinates. We know from Lemma 3.2 that $X$ is an upper triangular matrix with $X[k, k]=\left\langle N_{k-1}, C_{k-1}\right\rangle$. So $\operatorname{det}(X)=\prod_{k=1}^{i-1}\left\langle N_{k-1}, C_{k-1}\right\rangle$. The product $M X$ has $\left\langle C_{j}, N_{k}\right\rangle$ as its $(j, k)$ th element, so it is a lower triangular matrix by prefix orthogonality. Hence $\operatorname{det}(M X)$ is the product of its diagonal values, i.e, $\operatorname{det}(M X)=\prod_{k=1}^{i-1}\left\langle N_{k}, C_{k}\right\rangle$. Since $\operatorname{det}(M) \cdot \operatorname{det}(X)=\operatorname{det}(M X),\left\langle N_{0}, C_{0}\right\rangle=1$, and $\left\langle N_{k}, C_{k}\right\rangle \neq 0$ for all $k$, the lemma follows.

We know by Lemma 3.2 that $\left(x_{j, 1}, \ldots, x_{j, i-1}\right)$ is the unique solution to $M \mathbf{x}=b_{j}$. In the $i$ th iteration of the algorithm, we could have directly computed the vector $N_{i}=\left\langle N_{i-1}, C_{i-1}\right\rangle\left(x_{i, 1}, \ldots, x_{i, i-1}, 1,0, \ldots\right)$, which is $\operatorname{det}(M)\left(x_{i, 1}, \ldots, x_{i, i-1}, 1,0, \ldots\right)$ (by Lemma 3.3), by solving the set of equations $M \mathbf{x}=\operatorname{det}(M) b_{i}$ and appending $(\operatorname{det}(M), 0, \ldots, 0)$ to $\mathbf{x}$. However, such an algorithm would be slower-it would take time $\tilde{\Theta}\left(m^{\omega+2}\right)$, where $\omega<2.376$ is the exponent of matrix multiplication. The updates and normalizations in the algorithm deterministic-MCB achieve the same result in a more efficient manner. 
Let us now bound the running time of the $i$ th iteration of deterministic-MCB. We will show in section 7 that a shortest cycle $C_{i}$ such that $\left\langle C_{i}, N_{i}\right\rangle \neq 0$ can be computed in $O\left(m^{2} n+m n^{2} \log n\right)$ time. Let us look at bounding the time taken for the update and normalization steps. We take $O(m)$ arithmetic steps for updating and scaling each $N_{j}$ since each $N_{j}$ has $m$ coordinates. Thus the total number of arithmetic operations in the $i$ th iteration is $O((d-i) m)=O(m d)$ over all $j, i+1 \leq j \leq d$. We next estimate the cost of the arithmetic. The coordinates of $N_{j}$ are determined by the system $M \mathbf{x}=\operatorname{det}(M) b_{j}$ and hence are given by Cramer's rule. Fact 1 below shows that each entry in $N_{j}$ is bounded by $d^{d / 2}$. Thus we pay $\tilde{O}(d)$ time per arithmetic operation. Thus the running time of the $i$ th iteration is $\tilde{O}\left(\mathrm{~m}^{3}\right)$, and hence the running time of deterministic-MCB is $\tilde{O}\left(m^{4}\right)$.

FACT 1. Since $M$ is a $\pm 1,0$ matrix of size $(i-1) \times(i-1)$ and $b_{j}$ is a $\pm 1,0$ vector, all determinants used in Cramer's rule in solving $M \mathbf{x}=\operatorname{det}(M) b_{j}$ are bounded by $i^{i / 2}$ using Hadamard's inequality. Therefore, the absolute value of each entry in $N_{j}$ in the ith iteration, where $j \geq i$, is bounded by $i^{i / 2}$.

4. A faster scheme. The update and normalization steps form the bottleneck in the algorithm deterministic-MCB. We will reduce their cost from $\tilde{O}\left(m^{4}\right)$ to $\tilde{O}\left(m^{\omega+1}\right)$.

- First, we delay updates until after several new cycles have been computed. For instance, we update $N_{\lfloor d / 2\rfloor+1}, \ldots, N_{d}$ not after each new cycle but in bulk after all of $C_{1}, C_{2}, \ldots, C_{\lfloor d / 2\rfloor}$ are computed.

- Second, we use a fast matrix multiplication method to do the updates for all of $N_{\lfloor d / 2\rfloor+1}, \ldots, N_{d}$ together, and not individually as before.

The scheme. The faster deterministic algorithm starts with the same configuration for the $N_{i}$ 's as before, i.e., $N_{i}$ is initialized to the $i$ th unit vector, $1 \leq i \leq d$. It then executes three steps. First, it computes $C_{1}, \ldots, C_{\lfloor d / 2\rfloor}$ and $N_{1}, \ldots, N_{\lfloor d / 2\rfloor}$ recursively, leaving $N_{\lfloor d / 2\rfloor+1}, \ldots, N_{d}$ at their initial values. Second, it runs a bulk update step in which $N_{\lfloor d / 2\rfloor+1}, \ldots, N_{d}$ are modified so that they become orthogonal to $C_{1}, \ldots, C_{\lfloor d / 2\rfloor}$. And third, $C_{\lfloor d / 2\rfloor+1}, \ldots, C_{d}$ are computed recursively modifying $N_{\lfloor d / 2\rfloor+1}, \ldots, N_{d}$ in the process.

A crucial point to note about the second recursive call is that it modifies $N_{\lfloor d / 2\rfloor+1}$, $\ldots, N_{d}$ while ignoring $C_{1}, \ldots, C_{\lfloor d / 2\rfloor}$ and $N_{1}, \ldots, N_{\lfloor d / 2\rfloor}$; how then does it retain the orthogonality of $N_{\lfloor d / 2\rfloor+1}, \ldots, N_{d}$ with $C_{1}, \ldots, C_{\lfloor d / 2\rfloor}$ that we achieved in the bulk update step? The trick lies in the fact that whenever we update any $N_{j}$ in $\left\{N_{\lfloor d / 2\rfloor+1}, \ldots, N_{d}\right\}$ in the second recursive call, we do it as $N_{j}=\sum_{k=\lfloor d / 2\rfloor+1}^{d} \alpha_{k} N_{k}$, where $\alpha_{k} \in \mathbb{Q}$. That is, the updated $N_{j}$ is obtained as a rational linear combination of $N_{\lfloor d / 2\rfloor+1}, \ldots, N_{d}$. Since the bulk update step prior to the second recursive call ensures that $N_{\lfloor d / 2\rfloor+1}, \ldots, N_{d}$ are all orthogonal to $C_{1}, \ldots, C_{\lfloor d / 2\rfloor}$ at the beginning of this step, the updated $N_{j}$ 's remain orthogonal to $C_{1}, \ldots, C_{\lfloor d / 2\rfloor}$. This property allows the second recursive call to work strictly in the bottom half of the data without looking at the top half.

The base case for the recursion is a subproblem of size 1 (let this subproblem involve $C_{\ell}, N_{\ell}$ ) in which case the algorithm simply retains $N_{\ell}$ as it is and computes $C_{\ell}$ using the algorithm in section 7 . With regards to time complexity, the bulk update step will be shown to take $O\left(m d^{\omega-1}\right)$ arithmetic operations.

We describe the bulk update procedure in the recursive call that computes the cycles $C_{\ell}, \ldots, C_{h}$ for some $h$ and $\ell$ with $h>\ell$. This recursive call works with the vectors $N_{\ell}, \ldots, N_{h}$ : all of these vectors are already orthogonal to $C_{1}, \ldots, C_{\ell-1}$. The recursive call runs as follows:

Copyright (C) by SIAM. Unauthorized reproduction of this article is prohibited. 
1. Compute the cycles $C_{\ell}, \ldots, C_{m i d}$, where mid $=\lceil(\ell+h) / 2\rceil-1$, using the vectors $N_{\ell}, \ldots, N_{\text {mid }}$ recursively.

2. Modify $N_{m i d+1}, \ldots, N_{h}$, which are untouched by the first step, to make them orthogonal to $C_{\ell}, \ldots, C_{m i d}$.

3. Compute $C_{m i d+1}, \ldots, C_{h}$ using these $N_{m i d+1}, \ldots, N_{h}$ recursively.

Step 2 is the bulk update step. We wish to update each $N_{j}$, mid $+1 \leq j \leq h$, to a rational linear combination of $N_{\ell}, \ldots, N_{m i d}$ and $N_{j}$ as follows: ${ }^{4}$

$$
N_{j}=\frac{\left\langle N_{m i d}, C_{m i d}\right\rangle}{\left\langle N_{\ell-1}, C_{\ell-1}\right\rangle} N_{j}+\sum_{t=\ell}^{m i d} \alpha_{t j} N_{t},
$$

where the $\alpha_{t j}$ 's are to be determined in a way which ensures that $N_{j}$ becomes orthogonal to $C_{\ell}, \ldots, C_{m i d}$. That is, for all $i, j$, where $\ell \leq i \leq$ mid and mid $+1 \leq j \leq h$, we want

$$
\frac{\left\langle N_{m i d}, C_{m i d}\right\rangle}{\left\langle N_{\ell-1}, C_{\ell-1}\right\rangle}\left\langle C_{i}, N_{j}\right\rangle+\sum_{t=\ell}^{m i d} \alpha_{t j}\left\langle C_{i}, N_{t}\right\rangle=0 .
$$

Rewriting the above relations in matrix form, we get

$$
A \cdot \mathcal{N}_{d} \cdot D=-A \cdot \mathcal{N}_{u} \cdot X
$$

where (let $k=$ mid $-\ell+1)$

- $A$ is a $k * m$ matrix, the $i$ th row of which is $C_{\ell+i-1}$,

- $\mathcal{N}_{d}$ is an $m *(h-k)$ matrix, the $j$ th column of which is $N_{m i d+j}$,

- $D$ is an $(h-k) *(h-k)$ scalar matrix with $\left\langle N_{\text {mid }}, C_{m i d}\right\rangle /\left\langle N_{\ell-1}, C_{\ell-1}\right\rangle$ in the diagonal,

- $\mathcal{N}_{u}$ is an $m * k$ matrix, the $t$ th column of which is $N_{\ell+t-1}$, and

- $X$ is the $k *(h-k)$ matrix of variables $\alpha_{t j}$, with $t$ indexing the rows and $j$ indexing the columns.

To compute the $\alpha_{t j}$ 's, we solve for $X=-\left(A \cdot \mathcal{N}_{u}\right)^{-1} \cdot A \cdot \mathcal{N}_{d} \cdot D$. Using fast matrix multiplication, we can compute $A \cdot \mathcal{N}_{u}$ and $A \cdot \mathcal{N}_{d}$ in $O\left(m k^{\omega-1}\right)$ time by splitting the matrices into $d / k$ square blocks and using fast matrix multiplication to multiply the blocks. Multiplying each element of $A \cdot \mathcal{N}_{d}$ with the scalar $\left\langle N_{\text {mid }}, C_{m i d}\right\rangle /\left\langle N_{\ell-1}, C_{\ell-1}\right\rangle$ gives us $A \cdot \mathcal{N}_{d} \cdot D$. Thus we compute the matrix $A \cdot \mathcal{N}_{d} \cdot D$ with $O\left(m k^{\omega-1}\right)$ arithmetic operations. Next, we find the inverse of $A \cdot \mathcal{N}_{u}$ with $O\left(k^{\omega}\right)$ arithmetic operations (this inverse exists because $A \cdot \mathcal{N}_{u}$ is a lower triangular matrix whose diagonal entries are $\left.\left\langle C_{i}, N_{i}\right\rangle \neq 0\right)$. Then we multiply $\left(A \cdot \mathcal{N}_{u}\right)^{-1}$ with $A \cdot \mathcal{N}_{d} \cdot D$ using $O\left(k^{\omega}\right)$ arithmetic operations. Thus we obtain $X$. Finally, we obtain $N_{m i d+1}, \ldots, N_{d}$ from $X$ using the product $\mathcal{N}_{u} \cdot X$, which we can compute in $O\left(m k^{\omega-1}\right)$ arithmetic operations, and adding $\mathcal{N}_{d} \cdot D$ to $\mathcal{N}_{u} \cdot X$. The total number of arithmetic operations required for the bulk update step is thus $O\left(m k^{\omega-1}\right)$.

The total number of arithmetic operations required for the bulk update step is $O\left(m k^{\omega-1}\right)$; however, each arithmetic operation is quite expensive since we deal with large numbers here. In this algorithm, the entries in $\left(A \cdot \mathcal{N}_{u}\right)^{-1}$ could be very large. The elements in $A \cdot \mathcal{N}_{u}$ have values up to $d^{\Theta(d)}$, which would result in the entries in $\left(A \cdot \mathcal{N}_{u}\right)^{-1}$ being as large as $d^{\Theta\left(d^{2}\right)}$. So each arithmetic operation then costs us

\footnotetext{
${ }^{4}$ Note that the coefficient $\left\langle N_{m i d}, C_{m i d}\right\rangle /\left\langle N_{\ell-1}, C_{\ell-1}\right\rangle$ for $N_{j}$ is chosen so that the updated vector $N_{j}$ here is exactly the same vector $N_{j}$ that we would have obtained at this stage using the algorithm deterministic-MCB (section 3).
} 
up to $\tilde{\Theta}\left(d^{2}\right)$ time, and the overall time for the outermost bulk update step would be $\tilde{\Theta}\left(m^{\omega+2}\right)$ time, which makes this approach slower than the algorithm deterministicMCB.

5. A fast deterministic algorithm. In the approach described in the previous section, we saw that entries in the matrix $\left(A \cdot \mathcal{N}_{u}\right)^{-1}$ (refer to (4.2)) could be as large as $d^{\Theta\left(d^{2}\right)}$. Thus we were not able to use the faster scheme for updating the vectors $N_{j}$, since each arithmetic operation could cost us up to $\tilde{\Theta}\left(d^{2}\right)$ time. But observe that the $\alpha_{t j}$ 's are just intermediate numbers in our computation. That is, they are the coefficients in

$$
\sum_{t=\ell}^{m i d} \alpha_{t j} N_{t}+\frac{\left\langle N_{m i d}, C_{m i d}\right\rangle}{\left\langle N_{\ell-1}, C_{\ell-1}\right\rangle} N_{j} .
$$

Our final aim is to determine the updated coordinates of $N_{j}$ which are at most $d^{d / 2}$ (see Fact 1$)$, since we know $N_{j}=\left(y_{1}, \ldots, y_{\text {mid }}, 0, \ldots, 0,\left\langle N_{\text {mid }}, C_{\text {mid }}\right\rangle, 0, \ldots, 0\right)$, where $\mathbf{y}=\left(y_{1}, \ldots, y_{\text {mid }}\right)$ is the solution to the linear system: $M \mathbf{y}=\operatorname{det}(M) b_{j} ; M$ is the mid $\times$ mid matrix of $C_{1}, \ldots, C_{m i d}$ truncated to the their first mid coordinates, and $b_{j}$ is the column vector of negated $j$ coordinates of $C_{1}, \ldots, C_{m i d}$.

Since the final coordinates are bounded by $d^{d / 2}$ while the intermediate values could be much larger, this suggests the use of modular arithmetic here. We could work over the finite fields $\mathbb{Z}_{p_{1}}, \mathbb{Z}_{p_{2}}, \ldots, \mathbb{Z}_{p_{s}}$ where $p_{1}, \ldots, p_{s}$ are small primes (say, in the range $d$ to $d^{2}$ ) and try to retrieve $N_{j}$ from $N_{j} \bmod p_{1}, \ldots, N_{j} \bmod p_{s}$, which is possible (by the Chinese remainder theorem) if $s \approx d / 2$. Arithmetic in $\mathbb{Z}_{p}$ takes $O(1)$ time and we thus spend $O\left(s \cdot m k^{\omega-1}\right)$ time for the update step now. However, if it is the case that some $p$ is a divisor of some $\left\langle N_{i}, C_{i}\right\rangle$ where $\ell \leq i \leq$ mid, then we cannot invert $A \cdot \mathcal{N}_{u}$ in the field $\mathbb{Z}_{p}$. Since each number $\left\langle N_{i}, C_{i}\right\rangle$ could be as large as $d^{d / 2}$, it could be a multiple of up to $\Theta(d)$ primes which are in the range $d, \ldots, d^{2}$. So in order to be able to determine $d$ primes which are relatively prime to each of $\left\langle N_{\ell}, C_{\ell}\right\rangle, \ldots,\left\langle N_{m i d}, C_{m i d}\right\rangle$, we might in the worst case have to test about $($ mid $-\ell+1) \cdot d=k d$ primes. Testing $k d$ primes for divisibility with respect to $k d$-bit numbers costs us $k^{2} d^{2}$ time. We cannot afford so much time per update step.

Another idea is to work over just one finite field $\mathbb{Z}_{q}$ where $q$ is a large prime. If $q>d^{d / 2}$, then $q$ can never be a divisor of any $\left\langle N_{i}, C_{i}\right\rangle$, so we can carry out all of our arithmetic in $\mathbb{Z}_{q}$ since the matrix $A \cdot \mathcal{N}_{u}$ will be nonsingular in $\mathbb{Z}_{q}$. Arithmetic in $\mathbb{Z}_{q}$ costs us $\tilde{\Theta}(d)$ time if $q \approx d^{d}$. Then our update step takes $\tilde{O}\left(m^{2} k^{\omega-1}\right)$ time, which will result in a total time of $\tilde{O}\left(m^{\omega+1}\right)$ for all of the update steps, which is our goal. But computing such a large prime $q$ is a difficult problem.

The solution is to work over a suitable ring instead of over a field; recall that fast matrix multiplication algorithms work over rings. Let us do the above computation modulo a large integer $R$, say, $R \approx d^{d}$. Then intermediate numbers do not grow more than $R$ and we can retrieve $N_{j}$ directly from $N_{j} \bmod R$, because $R$ is much larger than any coordinate of $N_{j}$.

What properties of $R$ do we need? The integer $R$ must be relatively prime to the numbers: $\left\langle N_{\ell}, C_{\ell}\right\rangle,\left\langle N_{\ell+1}, C_{\ell+1}\right\rangle, \ldots,\left\langle N_{m i d}, C_{m i d}\right\rangle$ so that the triangular matrix $A \cdot \mathcal{N}_{u}$ which has these elements along the diagonal is invertible in $\mathbb{Z}_{R}$. And $R$ must also be relatively prime to $\left\langle N_{\ell-1}, C_{\ell-1}\right\rangle$ so that $\left\langle N_{m i d}, C_{m i d}\right\rangle /\left\langle N_{\ell-1}, C_{\ell-1}\right\rangle$ is defined in $\mathbb{Z}_{R}$. Once we determine such an $R$, we will work in $\mathbb{Z}_{R}$. We stress the point that such an $R$ is a number used only in this particular bulk update step - in another bulk update step of another recursive call, we need to compute another such large integer. 
It is easy to see that the number $R$ determined below is a large number that is relatively prime to $\left\langle N_{\ell-1}, C_{\ell-1}\right\rangle,\left\langle N_{\ell}, C_{\ell}\right\rangle,\left\langle N_{\ell+1}, C_{\ell+1}\right\rangle, \ldots$, and $\left\langle N_{m i d}, C_{m i d}\right\rangle$ by doing the following.

1. Right at the beginning of the algorithm, compute $d^{2}$ primes $p_{1}, \ldots, p_{d^{2}}$, where each of these primes is at least $d$. Then form the $d$ products: $P_{1}=p_{1} \cdots p_{d}$, $P_{2}=p_{1} \cdots p_{2 d}, P_{3}=p_{1} \cdots p_{3 d}, \ldots, P_{d}=p_{1} \cdots p_{d^{2}}$.

2. Then during our current update step, compute the product

$$
\mathcal{L}=\left\langle N_{\ell-1}, C_{\ell-1}\right\rangle\left\langle N_{\ell}, C_{\ell}\right\rangle \cdots\left\langle N_{m i d}, C_{m i d}\right\rangle .
$$

3. By doing a binary search on $P_{1}, \ldots, P_{d}$, determine the smallest $s \geq 0$ such that $P_{s+1}$ does not divide $\mathcal{L}$.

4. Determine a $p \in\left\{p_{s d+1}, \ldots, p_{s d+d}\right\}$ that does not divide $\mathcal{L}$. Let $R=p^{d}$.

Cost of computing $R$. The value of $\pi(r)$, the number of primes less than $r$, is given by $r / 6 \log r \leq \pi(r) \leq 8 r / \log r$ [1]. So all the primes $p_{1}, \ldots, p_{d^{2}}$ are $\tilde{O}\left(d^{2}\right)$, and computing them takes $\tilde{O}\left(d^{2}\right)$ time using a sieving algorithm. The products $P_{1}, \ldots, P_{d}$ are computed just once in a preprocessing step. We will always perform arithmetic on large integers using Schönhage-Strassen multiplication, so that it takes $\tilde{O}(d)$ time to multiply two $d$-bit numbers. Whenever we perform a sequence of multiplications, we will use a tree so that $d$ numbers (each of bit size $\tilde{O}(d)$ ) can be multiplied in $\tilde{O}\left(d^{2}\right)$ time. So computing $P_{1}, \ldots, P_{d}$ takes $\tilde{O}\left(d^{3}\right)$ preprocessing time.

In the update step, we compute $\mathcal{L}$, which takes $\tilde{O}\left(d^{2}\right)$ time. The product $P_{s+1}=$ $p_{s d+1} \cdots p_{s d+d}$ is found in $\tilde{O}\left(d^{2}\right)$ time by binary search in the set $\left\{P_{1}, \ldots, P_{d}\right\}$. Determine a $p$ in the set $\left\{p_{s d+1}, \ldots, p_{s d+d}\right\}$ that does not divide $\mathcal{L}$ by testing which of the two products $p_{s d+1} \cdots p_{s d+\lfloor d / 2\rfloor}$ or $p_{s d+\lfloor d / 2\rfloor+1} \cdots p_{s d+d}$ does not divide $\mathcal{L}$, and recurse on the product that does not divide $\mathcal{L}$. Thus $R$ can be computed in $\tilde{O}\left(d^{2}\right)$ time.

Computation in $\mathbb{Z}_{R}$. We need to invert the matrix $A \cdot \mathcal{N}_{u}$ in the $\operatorname{ring} \mathbb{Z}_{R}$. Recall that this matrix is lower triangular. Computing the inverse of a lower triangular matrix is easy. If

$$
A \cdot \mathcal{N}_{u}=\left(\begin{array}{cc}
W & 0 \\
Y & Z
\end{array}\right) \text {, then we have }\left(A \cdot \mathcal{N}_{u}\right)^{-1}=\left(\begin{array}{cc}
W^{-1} & 0 \\
-Z^{-1} Y W^{-1} & Z^{-1}
\end{array}\right) .
$$

Hence to invert $A \cdot \mathcal{N}_{u}$ in $\mathbb{Z}_{R}$ we need the multiplicative inverses of only its diagonal elements: $\left\langle C_{\ell}, N_{\ell}\right\rangle, \ldots,\left\langle C_{m i d}, N_{\text {mid }}\right\rangle$ in $\mathbb{Z}_{R}$. Using Euclid's greatest common divisor (gcd) algorithm, each inverse can be computed in $\tilde{O}\left(d^{2}\right)$ time, since each of the numbers involved here and $R$ have bit size $\tilde{O}(d)$. The matrix $A \cdot \mathcal{N}_{u}$ is inverted via fast matrix multiplication, and once we compute $\left(A \cdot \mathcal{N}_{u}\right)^{-1}$, the matrix $X$, that consists of all the coordinates $\alpha_{t j}$ that we need (see (4.1)), can be easily computed in $\mathbb{Z}_{R}$ as $-\left(A \cdot \mathcal{N}_{u}\right)^{-1} \cdot A \cdot \mathcal{N}_{d} \cdot D$ by fast matrix multiplication. Then we determine all $N_{j} \bmod R$ for mid $+1 \leq j \leq h$ from $\mathcal{N}_{u} \cdot X+\mathcal{N}_{d} \cdot D$. It follows from the analysis presented in section 4 that the time required for all of these operations is $\tilde{O}\left(m^{2} k^{\omega-1}\right)$ since each number is now bounded by $d^{d}$.

Retrieving the actual $N_{j}$. Each entry of $N_{j}$ can have absolute value at most $d^{d / 2}$ (from Fact 1 ). The number $R$ is much larger than this, $R>d^{d}$. So if any coordinate, say, $n_{l}$ in $N_{j} \bmod R$, is larger than $d^{d / 2}$, then we can retrieve the original $n_{l}$ as $n_{l}-R$. Thus we can retrieve $N_{j}$ from $N_{j} \bmod R$ in $O\left(d^{2}\right)$ time. The time complexity for the update step, which includes matrix operations, gcd computations, and other arithmetic, is $\tilde{O}\left(m^{2} k^{\omega-1}+d^{2} k\right)$ or $\tilde{O}\left(m^{2} k^{\omega-1}\right)$. Thus our recurrence becomes 
(assuming Lemma 7.4 from section 7, which shows that the base case $T(1)$ takes $O\left(m^{2} n+m n^{2} \log n\right)$ time $)$

$$
T(k)= \begin{cases}2 T(k / 2)+\tilde{O}\left(m^{2} k^{\omega-1}\right) & \text { if } k>1, \\ m^{2} n+m n^{2} \log n & \text { if } k=1 .\end{cases}
$$

The recurrence solves to $T(k)=O\left(k\left(m^{2} n+m n^{2} \log n\right)+k^{\omega-1} m^{2} \cdot \operatorname{poly}(\log m)\right)$, and hence $T(d)=O\left(m^{3} n+m^{2} n^{2} \log n\right)+\tilde{O}\left(m^{\omega+1}\right)$, which is $O\left(m^{3} n+m^{2} n^{2} \log n\right)$, because $m \leq n^{2}$ implies $\tilde{O}\left(m^{\omega+1}\right)$ is always $o\left(m^{3} n\right)$. Thus we have shown the following theorem.

THEOREM 5.1. A minimum cycle basis in a weighted directed graph with $m$ edges and $n$ vertices and nonnegative edge weights can be computed in $O\left(m^{3} n+m^{2} n^{2} \log n\right)$ time.

6. Faster arithmetic via randomization. Suppose we could perform each arithmetic operation in $O(1)$ time; then our recurrence relation for $T(k), k>1$ will be given by:

$$
T(k)=2 T(k / 2)+O\left(m k^{\omega-1}\right) .
$$

We would also like to show that $T(1)$ is $O\left(m n+n^{2} \log n\right)$. Then this yields a running time of $O\left(n^{2} d \log n+n m d+m^{\omega}\right)$ or $O\left(m^{2} n+m n^{2} \log n\right)$, since $m^{\omega}$ is $o\left(m^{2} n\right)$. Such a running time matches the complexity of minimum cycle basis computation in undirected graphs.

Now we will show that we can perform each arithmetic operation in $O(1)$ time. This will require working modulo a randomly chosen prime $p$. We will perform all arithmetic over the finite field $\mathbb{Z}_{p}$ and not over the rationals. The danger now is that the results of working in this field could be different from those obtained by working over rationals. Fortunately, the following theorem claims that the results remain the same, provided the prime $p$ chosen satisfies certain properties. In the description below, let $C_{i}, N_{i}$ denote the results obtained by the fast deterministic algorithm working over the rationals, and let $C_{i}^{\prime}, N_{i}^{\prime}$ be the counterparts obtained by the fast deterministic algorithm working over $\mathbb{Z}_{p}$.

Theorem 6.1. If $\left\langle C_{i}, N_{i}\right\rangle \neq 0(\bmod p)$ for all $i, 1 \leq i \leq d$, then $C_{i}^{\prime}=C_{i}$ and $N_{i}^{\prime}=N_{i}(\bmod p)$.

Proof. Recall that the fast deterministic algorithm has a recursive structure. We use an inductive argument that mimics this recursion. At the very beginning, $N_{i}^{\prime}=N_{i}$ $(\bmod p)$ for all $i, 1 \leq i \leq d$, as the $N_{i}$ 's are $\{0,1\}$ vectors. Each recursive subproblem (when working with rationals) then takes a contiguous subset $N_{\ell}, N_{\ell+1}, \ldots, N_{h}$ and computes $C_{\ell}, C_{\ell+1}, \ldots, C_{h}$ using only $N_{\ell}, N_{\ell+1}, \ldots, N_{h}$, modifying the latter in the process. We claim that if this recursive subproblem began with $N_{\ell}^{\prime}, N_{\ell+1}^{\prime}, \ldots, N_{h}^{\prime}$ instead of $N_{\ell}, N_{\ell+1}, \ldots, N_{h}$, where

$$
N_{\ell}^{\prime}=N_{\ell}(\bmod p), N_{\ell+1}^{\prime}=N_{\ell+1}(\bmod p), \ldots, N_{h}^{\prime}=N_{h}(\bmod p),
$$

and subsequently worked modulo $p$, then it would still produce the same cycles $C_{\ell}, \ldots, C_{h}$ and in addition, after modification, the relations $N_{\ell}^{\prime}=N_{\ell}(\bmod p), N_{\ell+1}^{\prime}$ $=N_{\ell+1}(\bmod p), \ldots, N_{h}^{\prime}=N_{h}(\bmod p)$ will continue to hold. We will use induction on $\ell-h+1$ to prove this claim. We will explicitly show the base case when $\ell-h+1=1$. We will then assume that the claim is true for all recursive subproblems with $1 \leq \ell-h+1<t$ and then prove it for $\ell-h+1=t$. 
Consider a subproblem of size $1(\ell-h+1=1$, i.e., $\ell=h)$. When working over rationals, $N_{\ell}$ does not change and $C_{\ell}$ is defined as the shortest cycle such that $\left\langle C_{\ell}, N_{\ell}\right\rangle \neq 0$. When working $(\bmod p), N_{\ell}^{\prime}$ does not change and $C_{\ell}^{\prime}$ is defined as the shortest cycle such that $\left\langle C_{\ell}^{\prime}, N_{\ell}^{\prime}\right\rangle \neq 0(\bmod p)$. Assuming $N_{\ell}^{\prime}=N_{\ell}(\bmod p)$ at the beginning of this subproblem, and given that $p$ satisfies $\left\langle C_{\ell}, N_{\ell}\right\rangle \neq 0(\bmod p)$, it follows that $C_{\ell}^{\prime}=C_{\ell}$. This shows the base case.

Next, consider the three-step process used in the fast deterministic algorithm. First, consider the first recursive step which computes $C_{\ell}, \ldots, C_{m i d}$. By the induction hypothesis, at the end of this step, we have $C_{\ell}^{\prime}=C_{\ell}, \ldots, C_{m i d}^{\prime}=C_{m i d}$ and $N_{\ell}^{\prime}=N_{\ell}(\bmod p), \ldots, N_{m i d}^{\prime}=N_{m i d}(\bmod p)$. The vectors $N_{m i d+1}^{\prime}, \ldots, N_{h}^{\prime}$ are untouched by this step and by virtue of the initial assignment, stay identical to $N_{m i d+1}, \ldots, N_{h}$. Second, consider the bulk update step. This step modifies $N_{m i d+1}^{\prime} \ldots N_{h}^{\prime}$ as a function of $N_{\ell}^{\prime}, \ldots, N_{m i d}^{\prime}$ and $C_{\ell}^{\prime}, \ldots, C_{m i d}^{\prime}$. Since $N_{\ell}^{\prime}=$ $N_{\ell}(\bmod p), \ldots, N_{m i d}^{\prime}=N_{m i d}(\bmod p)$ and $C_{1}^{\prime}=C_{1}, \ldots, C_{m i d}^{\prime}=C_{m i d}$, it follows that $N_{\text {mid }+1}^{\prime}=N_{\text {mid }+1}(\bmod p), \ldots, N_{h}^{\prime}=N_{h}(\bmod p)$ after the bulk update step. This sets up the necessary initial condition for the second recursive step which computes $C_{m i d+1}^{\prime}, \ldots, C_{h}^{\prime}$ from $N_{m i d+1}^{\prime}, \ldots, N_{h}^{\prime}$ alone. Applying the induction hypothesis again proves the theorem.

Selecting the number $p$. It remains to show how $p$ is chosen. Consider a pool of $d^{2}$ primes, each of which is bigger than $d^{2}$, and suppose we choose a prime at random from this pool. Lemma 6.3 shows that it satisfies the conditions of Theorem 6.1 with probability of at least $3 / 4$. Let us first make the following definition.

Definition 6.2. Call a prime $p$ good if $\left\langle C_{i}, N_{i}\right\rangle \neq 0(\bmod p)$ for each $i \in$ $\{1, \ldots, d\}$. Call a prime $p$ bad if it is not good.

Lemma 6.3. Let $P$ be a set of $d^{2}$ primes, each of which is at least $d^{2}$. Then at least $3 / 4$ th of the set $P$ is good.

Proof. We will use Lemma 3.3 here. Lemma 3.3 shows that for all $1 \leq j \leq d$, $\left\langle C_{j}, N_{j}\right\rangle=\operatorname{det}(M)$, where $M$ is the $j \times j$ matrix of $\tilde{C}_{k}$ 's on the right-hand side of (3.1). Hadamard's inequality tells us that the absolute value of $\operatorname{det}(M)$ is at most $d^{d / 2}$, since the entries in $M$ are $0, \pm 1$. Hence for all $1 \leq i \leq d$, we have $0 \neq\left|\left\langle C_{i}, N_{i}\right\rangle\right| \leq d^{d / 2}$. Since each prime in $P$ is at least $d^{2}$, at most $d / 4$ elements in $P$ can be divisors of $\left\langle C_{i}, N_{i}\right\rangle$. So the number of primes in $P$ that can divide at least one of $\left\langle C_{1}, N_{1}\right\rangle,\left\langle C_{2}, N_{2}\right\rangle, \ldots,\left\langle C_{d}, N_{d}\right\rangle$ is at most $d^{2} / 4$. Hence the fraction of bad primes in $P$ is at most $d^{2} / 4 d^{2} \leq 1 / 4$.

We now need to show how to compute the pool $P$ of $d^{2}$ primes, each bigger than $d^{2}$. As mentioned earlier, the value of $\pi(r)$, the number of primes less than $r$, is given by $r / 6 \log r \leq \pi(r) \leq 8 r / \log r$. So the elements in $P$ can be bounded by $100 d^{2} \log d$. Using sieving, we can compute the set of primes in the first $100 d^{2} \log d$ numbers in $O\left(d^{2} \log ^{2} d\right)$ time. So the set $P$ can be determined in $O\left(d^{2} \log ^{2} d\right)=O\left(m^{2} \log ^{2} n\right)$ time. Note that this term does not appear in the final complexity of $O\left(m^{2} n+m n^{2} \log n\right)$ because it is completely dominated by the $m^{2} n$ term.

Arithmetic modulo $p$. Under the assumption that arithmetic on $O(\log m)$ bits takes unit time, it follows that addition, subtraction, and multiplication in $\mathbb{Z}_{p}$ can be implemented in unit time since $p$ is $O\left(d^{2} \log d\right)=O\left(m^{2} \log m\right)$. However, we also need to implement division efficiently. Once $p$ is chosen, we will compute the multiplicative inverses of all elements in $\mathbb{Z}_{p}^{*}$ by the extended Euclid's gcd algorithm by solving $a x=1(\bmod p)$ for each $a \in \mathbb{Z}_{p}^{*}$. This takes time $O(\log p)$ for each element and hence $O(p \log p)=O\left(d^{2} \log ^{2} d\right)$ for all of the elements. Thereafter, division in $\mathbb{Z}_{p}$ gets implemented as multiplication with the inverse of the divisor. The $O\left(d^{2} \log ^{2} d\right)$ 
term does not appear in the final complexity of $O\left(m^{2} n+m n^{2} \log n\right)$ because it is completely dominated by the $m^{2} n$ term.

Lemma 7.3, proved in section 7, shows that $T(1)$, the time taken to compute the shortest $C_{i}$ such that $\left\langle C_{i}, N_{i}\right\rangle \neq 0(\bmod p)$, is $O\left(m n+m n^{2} \log n\right)$. Hence Theorem 6.4 follows from the recurrence relation for $T(\cdot)$ (refer to the beginning of this section).

THEOREM 6.4. A minimum cycle basis in a directed graph with $n$ vertices and $m$ edges, with nonnegative weights on its edges, can be computed with a probability of at least $3 / 4$ in $O\left(m^{2} n+m n^{2} \log n\right)$ time.

7. Computing nonorthogonal shortest cycles. Now we come to the second key routine required by our algorithm - given a directed graph $G$ with nonnegative edge weights, compute a shortest cycle in $G$ whose dot product with a given vector $N_{i} \in \mathbb{Z}^{m}$ is nonzero. We will first consider the problem of computing a shortest cycle $C_{p}$ such that $\left\langle C_{p}, N_{i}\right\rangle \neq 0(\bmod p)$ for a number $p=O\left(d^{2} \log d\right)$. Recall that $C_{p}$ can traverse edges of $G$ in both forward and reverse directions; the vector representation of $C_{p}$ has a 1 for every forward edge in the cycle, a -1 for every reverse edge, and a 0 for edges not present at all in the cycle. This vector representation is used for computing dot products with $N_{i}$. The weight of $C_{p}$ itself is simply the sum of the weights of the edges in the cycle. We show how to compute $C_{p}$ in $O\left(m n+n^{2} \log n\right)$ time.

Definitions. To compute shortest paths and cycles, we will work with the undirected version of $G$. Directions will be used only to compute the residue class of a path or cycle, i.e., the dot product between the vector representation of this path or cycle and $N_{i}$ modulo $p$. Let $p_{u v}$ denote a shortest path between vertices $u$ and $v$ and let $f_{u v}$ denote its length and $r_{u v}$ its residue class. Let $s_{u v}$ be the length of a shortest path, if any, between $u$ and $v$ in a residue class distinct from $r_{u v}$. Observe that the value of $s_{u v}$ is independent of the choice of $p_{u v}$.

We will show how to compute $f_{u v}$ and $s_{u v}$ for all pairs of vertices $u, v$ in $O(m n+$ $\left.n^{2} \log n\right)$ time. As is standard, we will also compute paths realizing these lengths in addition to computing the lengths themselves. The following claim tells us how these paths can be used to compute a shortest nonorthogonal cycle - simply take each edge $u v$ and combine it with $s_{v u}$ to get a cycle. The shortest of all these cycles having a nonzero residue class is our required cycle.

LEMMA 7.1. Let $C=u_{0} u_{1} \ldots u_{k} u_{0}$ be a shortest cycle whose residue class is nonzero modulo $p$ and whose shortest edge is $u_{0} u_{1}$. Then the path $u_{1} u_{2} \ldots u_{k} u_{0}$ has a residue class different from the residue class of the edge $u_{1} u_{0}$, the length of the path $u_{1} u_{2} \ldots u_{k} u_{0}$ equals $s_{u_{1} u_{0}}$, and the length of the edge $u_{0} u_{1}$ equals $f_{u_{1} u_{0}}$.

Proof. First, we show that the path $u_{1} u_{2} \ldots u_{k} u_{0}$ and the edge $u_{1} u_{0}$ have different residue classes. Let $x$ denote the residue class of the path, and let $y$ denote the residue class of the edge $u_{0} u_{1}$. Since $C$ is in a nonzero residue class, $x+y \not \equiv 0(\bmod p)$, so $x \not \equiv-y(\bmod p)$. Since the incidence vector corresponding to $u_{1} u_{0}$ is the negation of the incidence vector corresponding to $u_{0} u_{1}$, the residue class of the edge $u_{1} u_{0}$ is $-y$. Thus the claim follows.

Now, if the length of $u_{1} u_{0}$ is strictly greater than $f_{u_{1} u_{0}}$, then consider any shortest path $\pi$ between $u_{1}$ and $u_{0}$ (which, of course, has length $f_{u_{1} u_{0}}$ ). Combining $\pi$ with $u_{1} u_{0}$ yields a cycle and combining $\pi$ with $u_{1} u_{2} \ldots u_{k} u_{0}$ yields another cycle. These cycles are in distinct residue classes and are shorter than $C$. This contradicts the definition of $C$. Therefore, the edge $u_{1} u_{0}$ has length $f_{u_{1} u_{0}}$.

Since $u_{1} u_{2} \ldots u_{k} u_{0}$ has a different residue class from the edge $u_{1} u_{0}$, the length of $u_{1} u_{2} \ldots u_{k} u_{0}$ cannot be smaller than $s_{u_{1} u_{0}}$, by the very definition of $s_{u_{1} u_{0}}$. Suppose, 
for a contradiction, that the length $u_{1} u_{2} \ldots u_{k} u_{0}$ is strictly larger than $s_{u_{1} u_{0}}$. Then combining the path between $u_{1}$ and $u_{0}$ which realizes the length $s_{u_{1} u_{0}}$ along with the edge $u_{1} u_{0}$ yields a cycle which is shorter than $C$ and which has a nonzero residue class modulo $p$. This contradicts the definition of $C$. The lemma follows.

Computing $f_{u v}$ and $s_{u v}$. We first find any one shortest path (among possibly many) between each pair of vertices $u$ and $v$ by Dijkstra's algorithm; this gives us $p_{u v}, f_{u v}$, and $r_{u v}$ for each pair $u, v$. The time taken is $O\left(m n+n^{2} \log n\right)$. For each pair $u, v$, we now need to find a shortest path between $u, v$ with a residue class distinct from $r_{u v}$; the length of this path will be $s_{u v}$. Use $q_{u v}$ to denote any such path. We show how a modified Dijkstra search can compute these paths in $O\left(m n+n^{2} \log n\right)$ time. The following lemma shows the key prefix property of the $q_{u v}$ paths needed for a Dijkstra-type algorithm.

Lemma 7.2. For any $u$ and $v$, the path $q_{u v}$ can be chosen from the set $\left\{p_{u w} \circ\right.$ $\left.w v, q_{u w} \circ w v: w v \in E\right\}$. Here $p \circ e$ denotes the path $p$ extended by the edge $e$.

Proof. Consider any path $\pi$ between $u$ and $v$ that realizes the value $s_{u v}$, i.e., it has length $s_{u v}$ and residue class distinct from $r_{u v}$. Let $w$ be the penultimate vertex on this path, and let $\pi^{\prime}$ be the prefix path from $u$ to $w$. Clearly, $\pi$ cannot be shorter than $p_{u w} \circ w v$. Hence, if the residue class of $p_{u w} \circ w v$ is distinct from $r_{u v}$, then we are done. So assume that $p_{u w} \circ w v$ has residue class $r_{u v}$. Then $\pi^{\prime}$ must have a residue class distinct from $p_{u w}$ and hence $q_{u w}$ exists. Also, the length of $\pi^{\prime}$ must be at least the length of $q_{u w}$, and the residue class of $q_{u w} \circ w v$ is distinct from the residue class of $p_{u w} \circ w v$ and hence distinct from $r_{u v}$. Thus $q_{u w} \circ w v$ realizes $s_{u v}$.

We now show how to compute the $s_{u v}$ 's for any fixed $u$ in time $O(m+n \log n)$ with a Dijkstra-type algorithm. Repeating this for every source gives the result. The algorithm differs from Dijkstra's shortest path algorithm only in the initialization and update steps, which we describe below. We use the notation $k e y_{u v}$ to denote the key used to organize the priority heap; keyuv will finally equal $s_{u v}$.

Initialization. We set $k e y_{u v}$ to the minimal length of any path $p_{u w} \circ w v$ with residue class distinct from $r_{u v}$. If there is no such path, then we set it to $\infty$.

The update step. Suppose we have just removed $w$ from the priority queue. We consider the $u-w$ path of length keyuw which was responsible for the current key value of $w$. For each edge $w v$ incident on $w$, we extend this path via the edge $w v$. We update keyuv to the length of this path provided its residue class is different from $r_{u v}$.

Correctness. We need to show that keyuv is set to $s_{u v}$ in the course of the algorithm (note that one does not need to worry about the residue class since any path that updates $k e y_{u v}$ in the course of the algorithm has residue class different from $r_{u v}$ ). This follows immediately from Lemma 7.2. If $s_{u v}$ is realized by the path $p_{u w} \circ w v$ for some neighbor $w$, then $k e y_{u v}$ is set to $s_{u v}$ in the initialization step. If $s_{u v}$ is realized by the path $q_{u w} \circ w v$ for some neighbor $w$, then $k e y_{u v}$ is set to $s_{u v}$ in the update step. This completes the proof of correctness. Thus we have given an $O\left(m n+n^{2} \log n\right)$ algorithm to compute the $s_{u v}$ 's for all $u, v \in V$. We have thus shown the following lemma.

Lemma 7.3. A shortest cycle $C_{p}$ in $G$, whose dot product with $N_{i}$ is nonzero modulo $p$, can be computed in $O\left(m n+n^{2} \log n\right)$ time.

We now consider the complexity of computing a shortest cycle $C_{i}$ whose dot product with $N_{i}$ is nonzero, instead of the condition that the dot product is nonzero modulo $p$. But any cycle $C_{i}$ which satisfies $\left\langle C_{i}, N_{i}\right\rangle \neq 0$ satisfies $\left\langle C_{i}, N_{i}\right\rangle \neq 0(\bmod p)$ for some $p \in\left\{p_{1}, \ldots, p_{d / 2}\right\}$, where $p_{1}, \ldots, p_{d / 2}$ are distinct primes, each of which is at least $d$. This follows from the isomorphism of the ring $\mathbb{Z}_{\prod p_{i}}$ to the ring $\mathbb{Z}_{p_{1}} \times$ 
$\mathbb{Z}_{p_{2}} \times \cdots \times \mathbb{Z}_{p_{d / 2}}$. So any nonzero element whose absolute value is less than $\prod_{i=1}^{d / 2} p_{i}$ is mapped to a tuple of its residues that is not the zero vector.

We have $\left|\left\langle C_{i}, N_{i}\right\rangle\right| \leq d^{d / 2}$ (from Lemma 3.3 and Hadamard's inequality). Thus $\left|\left\langle C_{i}, N_{i}\right\rangle\right|<\prod_{i=1}^{d / 2} p_{i}$. So if $\left\langle C_{i}, N_{i}\right\rangle$ is nonzero, then it is a nonzero element in $\mathbb{Z}_{\Pi p_{i}}$, and so it satisfies $\left\langle C_{i}, N_{i}\right\rangle \neq 0(\bmod p)$ for some $p$ in $\left\{p_{1}, \ldots, p_{d / 2}\right\}$. Thus a shortest cycle $C_{i}$ such that $\left\langle C_{i}, N_{i}\right\rangle \neq 0$ is the shortest among all the cycles $C_{p}$, $p \in\left\{p_{1}, \ldots, p_{d / 2}\right\}$, where $C_{p}$ is a shortest cycle such that $\left\langle C_{p}, N_{i}\right\rangle \neq 0(\bmod p)$. Hence, by Lemma 7.3, the time taken to compute $C$ is $O\left(d \cdot\left(m n+n^{2} \log n\right)\right)$ or $O\left(m^{2} n+m n^{2} \log n\right)$. Thus we have shown Lemma 7.4.

Lemma 7.4. A shortest cycle $C_{i}$ in $G$, whose dot product with $N_{i}$ is nonzero, can be computed in $O\left(m^{2} n+m n^{2} \log n\right)$ time.

8. Conclusions. We considered the minimum cycle basis problem in directed graphs with nonnegative edge weights. We presented an $O\left(m^{3} n+m^{2} n^{2} \log n\right)$ deterministic algorithm and an $O\left(m^{2} n+m n^{2} \log n\right)$ randomized algorithm for this problem, where $m$ is the number of edges and $n$ is the number of vertices. These algorithms use the framework of computing cycles $C_{1}, \ldots, C_{d}$ and their supporting vectors $N_{1}, \ldots, N_{d}$ using fast matrix multiplication. However, this approach leads to large intermediate numbers and the cost of arithmetic becomes high. We overcome this problem in the randomized algorithm by working over a finite field $\mathbb{Z}_{p}$ for a small random prime $p$ and by working over suitable rings $\mathbb{Z}_{R}$ in the deterministic algorithm. We also presented an efficient algorithm, based on Dijkstra's algorithm, to compute a shortest cycle whose dot product with a function on its edge set is nonzero.

\section{REFERENCES}

[1] T. M. Apostol, Introduction to Analytic Number Theory, Springer-Verlag, New York, 1997.

[2] C. Berge, Graphs, North-Holland, Amsterdam, The Netherlands, 1985.

[3] F. Berger, P. Gritzmann, and S. De Vries, Minimum cycle bases for network graphs, Algorithmica, 40 (2004), pp. 51-62.

[4] B. Bollobás, Modern Graph Theory, in Graduate Texts in Mathematics 184, Springer-Verlag, New York, 1998.

[5] A. C. Cassell, J. C. Henderson, and K. Ramachandran, Cycle bases of minimal measure for the structural analysis of skeletal structures by the flexibility method, Proc. Royal Society of London Series A, 350 (1976), pp. 61-70.

[6] T. F. Coleman and A. Pothen, The null space problem I. Complexity, SIAM J. Algebraic Discrete Methods, 7 (1986), pp. 527-537.

[7] T. F. Coleman And A. Pothen, The null space problem II. Algorithms, SIAM J. Algebraic Discrete Methods, 8 (1987), pp. 544-563.

[8] D. Coppersmith AND S. WinOGRAD, Matrix multiplications via arithmetic progressions, J. Symbolic Comput., 9 (1990), pp. 251-280.

[9] J. C. DE PInA, Applications of Shortest Path Methods, Ph.D. thesis, University of Amsterdam, Amsterdam, The Netherlands, 1995.

[10] N. Deo, Graph Theory with Applications to Engineering and Computer Science, Prentice-Hall Series in Automatic Computation, Prentice-Hall, Englewood Cliffs, NJ, 1982.

[11] P. M. Gleiss, Short Cycles: Minimum Cycle Bases of Graphs from Chemistry and Biochemistry, Ph.D. thesis, Universität Wien, Wein, Germany, 2001.

[12] P. M. Gleiss, J. Leydold, And P. F. Stadler, Circuit bases of strongly connected digraphs, Discuss. Math. Graph Theory, 23 (2003), pp. 241-260.

[13] A. Golynski And J. D. Horton, A polynomial time algorithm to find the minimum cycle basis of a regular matroid, in Proceedings of SWAT, Lecture Notes in Comput. Sci. 2368, Springer, Berlin, 2002, pp. 200-209.

[14] R. Hariharan, T. Kavitha, and K. Mehlhorn, A faster deterministic algorithm for minimum cycle basis in directed graphs, in Proceedings of ICALP, Lecture Notes in Comput. Sci. 4051, Springer, Berlin, 2006, pp. 250-261. 
[15] J. D. Horton, A polynomial-time algorithm to find a shortest cycle basis of a graph, SIAM J. Comput., 16 (1987), pp. 358-366.

[16] T. Kavitha, An $\tilde{O}\left(m^{2} n\right)$ randomized algorithm to compute a minimum cycle basis of a directed graph, in Proceedings of ICALP, Lecture Notes in Comput. Sci. 3580, Springer, Berlin, 2005, pp. 273-284.

[17] T. Kavitha and K. Mehlhorn, Algorithms to compute minimum cycle bases in directed graphs, Theory of Comput. Syst., 40 (2007), pp. 485-505.

[18] T. Kavitha, K. Mehlhorn, D. Michail, and K. Paluch, A faster algorithm for minimum cycle bases of graphs, in Proceedings of ICALP, Lecture Notes in Comput. Sci. 3142, Springer, Berlin, 2004, pp. 846-857.

[19] J. Leydold And P. F. Stadler, Minimal cycle bases of outerplanar graphs, Electron. J. Combin., 5 (1998), pp. 1-14.

[20] C. Liebchen, Finding short integral cycle bases for cyclic timetabling, in Proceedings of ESA, Lecture Notes in Comput. Sci. 2832, 2003, pp. 715-726.

[21] C. Liebchen and L. Peeters, On Cyclic Timetabling and Cycles in Graphs, Technical report 761/2002, TU Berlin, Berlin, Germany.

[22] C. Liebchen and R. Rizzi, A greedy approach to compute a minimum cycle basis of a directed graph, Inform. Process. Lett., 94 (2005), pp. 107-112.

[23] G. Tewari, C. Gotsman, and S. J. Gortler, Meshing genus-1 point clouds using discrete one-forms, Computers and Graphics, 30 (2006), pp. 917-926.

Copyright (C) by SIAM. Unauthorized reproduction of this article is prohibited. 